\title{
SOFT GRAVITONS SCREEN COUPLING IN DE SITTER SPACE
}

\author{
HIROYUKI KITAMOTO ${ }^{*, \ddagger}$ and YOSHIHISA KITAZAWA $*, \dagger, \S$ \\ * KEK Theory Center, \\ Tsukuba, Ibaraki 305-0801, Japan \\ † The Graduate University for Advanced Studies, \\ Department of Particle and Nuclear Physics, \\ Tsukuba, Ibaraki 305-0801, Japan \\ $\ddagger$ kitamoto@post.kek.jp \\ §itazawa@post.kek.jp
}

\begin{abstract}
On the de Sitter background, the gravitational propagator contains an infra-red logarithm term which breaks the de Sitter symmetry. We investigate how such soft gravitons affect the local dynamics of matter fields. We show that the infra-red logarithms do not spoil Lorentz invariance in scalar, Dirac and gauge field theories. In the interacting field theory with quartic, Yukawa and gauge interactions, we find that the effective couplings are time dependent and the evolutions are physical as their relative scaling exponents are gauge invariant.
\end{abstract}

Keywords: de Sitter space; super-horizon; graviton.

PACS Numbers: 04.60.-m, 04.62.+v, 95.36.+x

\section{Time Dependence Induced by Soft Gravitons}

In de Sitter ( $\mathrm{dS}$ ) space, the propagator of a massless and minimally coupled scalar field and gravitational field contains a de Sitter symmetry breaking term. This term is a direct consequence of the scale invariant spectrum and depends logarithmically on the scale of the universe. ${ }^{1}$ So in some field theoretic models in dS space, physical quantities acquire time dependences through the propagator.

For a general scalar field theory, we need to fine-tune the mass term to obtain such infra-red (IR) effects. On the other hand, the gravitational field contains massless and minimally coupled modes without the fine-tuning. In this regard, the gravitational field is an attractive candidate which induces the IR effects. ${ }^{2}$ The leading IR effect at the $n$-loop level manifests as $\left(G H^{2}\right)^{n} \log ^{n} a(t), a(t)=e^{H t}$. It indicates that the IR effects may grow up to certain values which are not suppressed by the gravitational constant.

We have investigated soft gravitational effects on the local dynamics of matter fields at the sub-horizon scale. ${ }^{3}$ Although we can not observe the super-horizon 
modes directly, it is possible that virtual gravitons of the super-horizon scale affect microscopic physics which are directly observable.

\section{Lorentz Invariance}

In investigating soft gravitational effects on local matter dynamics, it is a nontrivial question whether they preserve the Lorentz invariance. To verify that, we have investigated the IR effects on the kinetic terms of matter fields. As a result, we have found that soft gravitons do not spoil Lorentz invariance in scalar, Dirac and gauge field theories at the one-loop level. The IR logarithms can be absorbed by time dependent wave function renormalization factors and so soft gravitons do not contribute to free field theories.

Soft gravitational effects on free field theories have been investigated in a different parametrization of the metric. ${ }^{4,5}$ The interpretation of the difference due to the parametrization is under investigation.

\section{Screening of Couplings}

When some interaction terms exist in the action, the wave function renormalization and gravitons running between the vertexes contribute to the non-linear terms. As examples, we have adopted quartic, Yukawa and gauge interactions. These dimensionless couplings are dynamically screened by soft gravitons. Furthermore we have found that the time dependence of each coupling is gauge dependent but their relative scaling exponents are gauge invariant.

We interpret our findings as follows. The time dependence of each coupling is gauge dependent since there is no unique way to specify the time as it depends on an observer. A sensible strategy may be to pick a particular coupling and use its time evolution as a physical time.

\section{Acknowledgments}

We would like to thank Osaka City University and Nagoya University GCOE, which organized the conference "Progress in Quantum Field Theory and String Theory".

\section{References}

1. A. Vilenkin and L. H. Ford, Phys. Rev. D 26, 1231 (1982). A. D. Linde, Phys. Lett. B 116, 335 (1982). A. A. Starobinsky, Phys. Lett. B117, 175 (1982).

2. N. C. Tsamis and R. P. Woodard, Commun. Math. Phys. 162, 217 (1994).

3. H. Kitamoto and Y. Kitazawa, arXiv:1203.0391 [hep-th], arXiv:1204.2876 [hep-th].

4. E. O. Kahya and R. P. Woodard, Phys. Rev. D 76, 124005 (2007), Phys. Rev. D 77, 084012 (2008).

5. S. P. Miao and R. P. Woodard, Class. Quant. Grav. 23, 1721 (2006), Phys. Rev. D 74, 024021 (2006), Class. Quant. Grav. 25, 145009 (2008). 